\title{
LOW ENERGY ION REFLECTION FROM METAL SINGLE CRYSTALS
}

\author{
A. Van VEEN and J. HAAK \\ Fysisch Laboratorium der Rijksuniversiteit, Utrecht, The Netherlands
}

Received 4 April 1972

\begin{abstract}
Energy and angular distributions of ions reflected from metal single crystals have been computed. The neutralisation process is involved in the computing model. The result indicates a strong dependence on the surface trajectories of the reflected particles.
\end{abstract}

Until now there has been done very little work on the subject of reflection of low energetic ions from single crystal surfaces. The energy region of our interest ranges from 50 to $500 \mathrm{eV}$ ion energy. For higher energies many results are known (see reviews $[1,2]$ ). Also there is an extensive literature about scattering at thermal and hyperthermal energies.

The reflection in the energy region under consideration has his own specific character differentiating it from the neighbouring regions:

i. Thermal effects are of minor importance.

ii. Most of the ions reflect after collisions with atoms in the first and second layers of the crystal only.

iii. Violent collisions do not occur. As a consequence the sole inelastic processes to be considered are neutralisation processes.

It may be assumed that experimental problems have been overcome nowadays. Heiland and Taglauer (unpublished) have done experiments in this energy region. But theoretical treatment is complicated by the fact that the collision processes not always can be regarded as successive binary collisions.

So we have made a computer simulation of the reflection process to search for the specific features of the angular and energy distribution of the reflected particles. This is done for the case of Ar-ions reflected from $\mathrm{Ni}$ single crystals with different orientations. The energy of the ions is $300 \mathrm{eV}$ and the initial direction of the ions is always at an angle of 30 degrees with the surface, but lying in different planes of incidence.

The computing method involves solving of the equations of motion of the participating atoms and the ion by a multistep-method with an accuracy of the 4 th order. The timestep is gliding. The energy con- servation is within $1 \%$. The initial conditions are given by the velocity vector and the impactpoint of the ion, and the positions of the lattice atoms from which in. teraction with the incoming ion can be expected. Mostly it is sufficient to take $10-15$ atoms. The impact points of the ion are chosen in a representative area, defined by the symmetry of the crystal surface. The interaction potential $V(r)$ between the particles has been assumed to be of the Born-Mayer type: $V(r)=A \exp (--b r)$, where $r$ is the distance between the particles, $A$ and $b$ are constants depending on the ion-atom and atom-atom interaction, but independent of the charge state of the particles. Generally atomatom interactions can be neglected, when the ionatom mass ratio $<1$. The moving atoms do not interfere with the ion trajectory in that case.

The results show that the angular and energy distributions are similar to that of the reflection from an isolated atom chain, when the following conditions are fulfilled:

1. The tangential component of the injection direction is parallel with atom chains in the surface.

2. The atoms of different parallel chains are lying exactly side by side.

A characteristic example is given in fig. 1 (parts 3,4) where the reflection data for ions impinging on an isolated chain fit the data for the whole surface (in practice: 3 atom rows) very well.

For an isolated chain the scattering angle is limited between a minimum and a maximum scattering angle ( $\alpha \min , \alpha \max$ ), due to the fact that pure single or double collision events cannot occur. Before and after such collisions there is a weak interaction with other atoms of the row. This explains too why the lower part of the curve ("single" collisions) is in- 


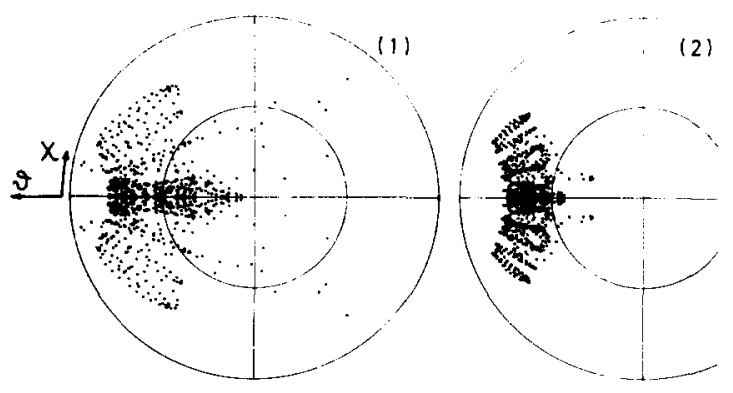

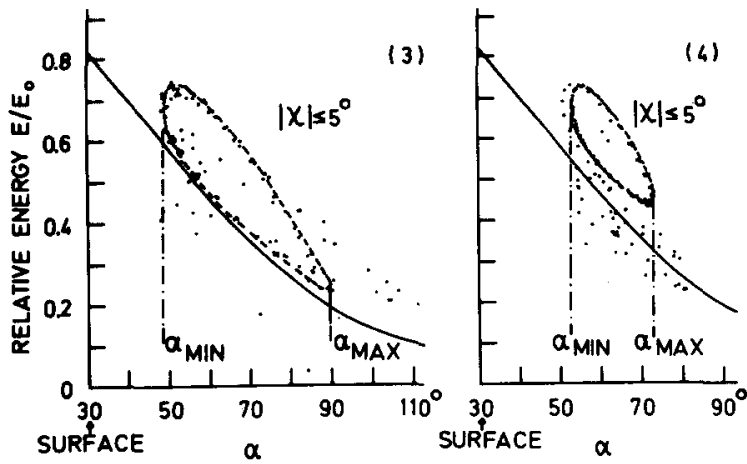

Fig. 1. Reflection of $300 \mathrm{eV} \mathrm{Ar}$ from Ni (110). The direction of incidence is lying in a $\{100\}$ plane perpendicular to the surface at an angle of $30^{\circ}$ with the surface plane. Born-Mayer potential parameters: $A=9 \times 10^{-15} \mathrm{~J}, b=6.1 \times 10^{+10} \mathrm{~m}^{-1}$ for (1) and (3); $A=9 \times 10^{-15} \mathrm{~J}, b=5.1 \times 10^{+10} \mathrm{~m}^{-1}$ for (2) and (4). (1) and (2): Spatial distributions of the reflected particles, polar angle $\theta$ varies from $0^{\circ}$ in the center to $90^{\circ}$ at the ou termost circle (linear scale), the azimuthal angle $x$ from $0^{\circ}-360^{\circ}$. (3) and (4): Relative energy $E / E_{\text {o }}$ versus polar scattering angle $\alpha(=120-\theta)$, for $|x| \leqslant 5^{\circ}$. Continuous curve: reflection from one atom (binary scattering). Dotted curve:

from a (110) chain. Black dots: from the (110) surface.

creased to an energy higher than can be expected from binary collision theory.

The case of an isolated chain differs from that for the whole surface.

i. An extra contribution of ions, focussed in the forward direction. The ion moves in a so-called "semichannel". A similar effect at higher energy has been shown by Yurasova [3].

ii. When the depth to width ratio of the channel is small the chains in the second layer contribute to the reflection, mainly in the forward direction (for instance: $(110\rangle$ chains in a (110) surface, fig. 1 parts 1,2).

When the above-mentioned condition number 2 is not fulfilled cooperation between chains does not occur. The reflection pattern appears to be disordered, however with a tendency to scatter in azimuthal directions where that condition does apply (for instance: $\langle 100\rangle$ chains in a (100) surface).

The influence of the interaction potential has been investigated by varying the potential parameters.

Larger effective diameters of the ions, realised by the choice of smaller $b$ values, result in

i. increasing $\alpha_{\text {min }}$ and decreasing $\alpha_{\text {max }}$,

ii. larger deviations from the binary scattering,

iii. increasing focussing effects.

Comparison of the results from these computations with the results from measurements may be difficult. Most of the measurements are done for reflected particles in ionised state. However, the mean probability to be in ionised state after reflection is very low $\left(\approx 10^{-4}\right)$ [4]. That probability depends on the specific trajectory of a reflected particle.

To incorporate neutralisation via Auger processes or eventually resonance processes [5], simulations have been made by solving the equations of motion simultaneously with the differential equation describing the neutralisation. The equation used is

$\mathrm{d} \ln P(t) / \mathrm{d} t=-C \sum_{i=1}^{N} \exp \left(-a s_{i}\right)$,

with $P(t)=$ probability to be in ionised state at time $t, P(0)=1, C=$ transition rate, $a=$ constant, $s_{i}=$ distance between atom number $i$ and the ion, $N=$ number of lattice atoms. This neutralisation equation is proposed to preserve the atomic character of the surface during the neutralisation process, in ccntrast to the flat potential model commonly used.

The preliminary conclusions from these computations are for $C=3.4 \times 10^{15} \mathrm{~s}^{-1}$ and $a=2 \times 10^{+10}$ $\mathrm{m}^{-1}$ :

i. $P(\infty)$ is generally a factor three larger for single collisions than for double collisions.

ii. Particles scattered on chains lying in the second layer have a much smaller probability $P(\infty)$ than particles scattered on atoms in the surface. Reflection from adsorbed metal atoms on the surface still gives a much larger probability $P(\infty)$ to be in ionised state. These differences will give a distinction between spatial and energy distribution of reflected particles in neutralised and ionised state (for instance shifts in the 
positions of the maxima in the energy distributions). However, when it is possible to measure also the neutral component of the reflected particles valuable information comes available concerning both, the interaction potential parameters and the parameters describing the neutralisation process.

\section{References}

[1] U.A. Arifov, Interaction of atomic particles with a solid surface (Consultants Bureau, New York - London, 1969).

[2] D.G. Armour and G. Carter, Sci. Instr. 5 (1972) 2.

[3] V.E. Yurasova, International conference on Atomic collisions in solids, Norway (1971), to be published.

[4] H.D. Hagstrum, Phys. Rev. 123 (1961) 758.

[5] W.F. van der Weg and D.J. Bierman, Physica 44 (1969) 177. 